\title{
The Projection Problem for Predicates of Taste*
}

\author{
Dilip Ninan \\ Tufts University
}

\begin{abstract}
Utterances of simple sentences containing taste predicates (e.g. delicious, fun, frightening) typically imply that the speaker has had a particular sort of firsthand experience with the object of predication. For example, an utterance of The carrot cake is delicious would typically imply that the speaker had actually tasted the cake in question, and is not, for example, merely basing her judgment on the testimony of others. According to one approach, this acquaintance inference is essentially an implicature, one generated by the Maxim of Quality together with a certain principle concerning the epistemology of taste (Ninan 2014). We first discuss some problems for this approach that arise in connection with disjunction and generalized quantifiers. Then, after stating a conjecture concerning which operators 'obviate' the acquaintance inference and which do not, we build on Anand \& Korotkova 2018 and Willer \& Kennedy Forthcoming by developing a theory that treats the acquaintance requirement as a presupposition, albeit one that can be obviated by certain operators.
\end{abstract}

Keywords: predicates of taste, acquaintance inference, presupposition projection, supervaluationism, generalized quantifiers, modals, attitude verbs

\section{Introduction}

Utterances of simple sentences containing a predicate of taste typically give rise to an acquaintance inference: they typically convey that the speaker has a certain sort of first-hand experience with the object of predication (Mothersill 1984; Robson 2012; Pearson 2013; MacFarlane 2014; Ninan 2014; Klecha 2014; Bylinina 2017). For example, an utterance of (1) would normally suggest that the speaker has tasted the cake in question, an utterance of (2) would normally suggest that speaker had traveled with Mary before, and an utterance of (3) would normally suggest that the speaker had seen the movie in question:

* Thanks to audiences at SALT 30 and the New York Philosophy of Language Workshop. Thanks also to Chris Barker, Liz Coppock, Hans Kamp, Chris Kennedy, Arc Kocurek, Matt Mandelkern, Sarah Murray, Craige Roberts, Rachel Rudolph, Paolo Santorio, and Malte Willer. This essay was completed while I was a fellow at the Paris Institute for Advanced Study; I am grateful to the Institute and its staff for their support. 
(1) The carrot cake is delicious.

$\hookrightarrow$ the speaker has tasted the cake

(2) Traveling with Mary is fun.

$\hookrightarrow$ the speaker has traveled with Mary

(3) That movie is frightening.

$\hookrightarrow$ the speaker has seen the movie

If one had not tasted the carrot cake, but had merely heard that it was good, it would, for example, be better to say something like:

(4) Apparently, the carrot cake is delicious.

in place of (1). Taste predications contrast here with more 'factual' predications: if I were to say (5), for example, you wouldn't necessarily reach any very specific conclusion about the grounds for my assertion:

(5) The carrot cake contains gluten.

I might have learned that the cake contained gluten via testimony from a reliable source, or I might know this because I made the cake, or I might know this because I tasted it and am very sensitive to gluten.

The acquaintance inference is a default inference: in some situations an utterance of a simple taste sentence doesn't seem to imply that the speaker has the requisite sort of first-hand experience. One source of defeasibility arises with 'exocentric' uses of taste predicates. Typically, one uses a taste predicate to convey something about one's own tastes and sensibilities; these are autocentric uses. But sometimes we use taste predicates to convey something about someone else's tastes and sensibilities; these are exocentric uses (Lasersohn 2005: §6.1). Exocentric uses don't give rise to a speaker acquaintance inference, but they may give rise to some sort of acquaintance inference (Anand \& Korotkova 2018: 63). Consider, for example, the following dialogue.

a. $[A]$ : How is Mary's trip to Morocco going?

b. $[B]$ : It's great. The food is delicious, and she's met a lot of interesting people.

$\leftrightarrow B$ has tasted the food in Morocco

$\hookrightarrow$ Mary has tasted the food in Morocco

There may be other sources of defeasibility (see Pearson 2013 for some discussion of these), and there seems to be some speaker variation about how easily defeated the inference is. Although in what follows I will mostly be setting aside exocentric cases along with other sources of defeasibility, the theory offered in $\S 4$ is 
intended to accommodate such cases. And while taste predicates are not the only type of predicates that give rise to an acquaintance inference (see Pearson 2013: 16 and Ninan 2014: 291 for some other examples), we focus, for simplicity, on predicates of gustatory taste (e.g. delicious, tasty).

What is the status of the acquaintance inference? Is it an entailment, a presupposition, an implicature? Pearson (2013) analyzes the acquaintance inference as a presuppositional inference, and a few initial observations support this hypothesis. For example, like presuppositions - and unlike standard entailments - the acquaintance inference projects over negation. Compare delicious with the presupposition trigger stops:

(7) a. Mary stopped smoking.

b. Mary hasn't stopped smoking.

$\hookrightarrow$ Mary smoked in the past

(8) a. The carrot cake is delicious.

b. The carrot cake is not delicious-it isn't moist enough.

$\hookrightarrow$ the speaker has tasted the cake

And like presuppositions - and unlike standard conversational implicatures-the acquaintance inference is difficult to cancel in positive environments:

(9) \# Mary stopped smoking, but she never smoked in the past.

(10) ? The carrot cake is delicious, but I haven't tasted it.

But once we widen our gaze a bit, the projection profile of the acquaintance inference starts to look rather different from that of standard presuppositions. For example, while standard presuppositions project over epistemic modals, out of the antecedents of indicative conditionals, and out of questions, the acquaintance inference does not seem to project out of these environments (Pearson 2013; Ninan 2014):

(11) a. Mary must have stopped smoking.

b. Mary might have stopped smoking.

c. If Mary stopped smoking, her doctor will be pleased.

d. Did Mary stop smoking?

$\hookrightarrow$ Mary smoked in the past

(12) a. The carrot cake must have been delicious.

b. The carrot cake might have been delicious.

c. If the carrot cake was delicious, Mary will be pleased. 


\section{d. Was the carrot cake delicious?}

\section{$\hookrightarrow$ the speaker has tasted the cake}

It's worth observing that the acquaintance requirement is not even locally accommodated in these environments (Ninan 2014: 299-300). Sentence (12a), for example, does not mean it must be that: the speaker has tasted the carrot cake and found it delicious. In (12a), the acquaintance requirement doesn't project nor is it interpreted within the scope of the modal; it seems to simply disappear.

What are we to make of this? In earlier work, I suggested that the foregoing data supported the epistemic view, the hypothesis that the acquaintance inference is an implicature generated by the Maxim of Quality together with a principle concerning the epistemology of taste (Ninan 2014). An alternative view, one taken in different ways by Anand \& Korotkova (2018) and Willer \& Kennedy (Forthcoming), is to retain the idea that the acquaintance inference is a presuppositional inference, but then posit a mechanism that allows certain operators (e.g. epistemic modals) to erase any acquaintance content in their scope. We might term this the presupposition-plusobviation view. ${ }^{1}$

The aim of the present essay is to assess the debate between these two views while also gaining a broader view of the relevant empirical terrain. We begin in $\S 2$ by examining how these two views fare once we examine a broader set of projection data. The data here-data involving disjunction and generalized quantifiersappears to favor the presupposition-plus-obviation view over the epistemic view. In $\S 3$, we step back to examine an emerging pattern: it seems that while intensional operators obviate the acquaintance requirement (Klecha 2014; Cariani Forthcoming), extensional operators do not. In $\S 4$, we respond to these data by developing a version of the presupposition-plus-obviation view that has two main components: (1) a supervaluationist theory of projection, and (2) a mechanism which allows certain operators to obviate the acquaintance requirement. Among other things, the theory illuminates the interaction between the acquaintance requirement and various generalized quantifiers. In $§ 5$, we examine some further evidence-evidence concerning attitude verbs and disjunction — that bears on the truth of our conjecture concerning which operators obviate the acquaintance requirement and which do not.

1 While Anand \& Korotkova (2018) describe their view in more or less these terms, Willer \& Kennedy (Forthcoming) place more emphasis on the expressivist aspect of their proposal. But in their dynamic setting, no update is defined for a simple taste sentence (or the negation thereof) if the speaker hasn't tasted the item in question; this requirement is then lifted in the scope of certain operators (e.g. epistemic modals), and this effect is due to the lexical entries of those operators (Willer \& Kennedy Forthcoming: §3.2). That looks like presupposition plus obviation. That said, their view is rather different from Anand and Korotkova's, both in terms of philosophical motivation and in terms of formal implementation. 
The Projection Problem for Predicates of Taste

\section{The epistemic view}

The epistemic view consists of two principal claims. The first is simply the Maxim of Quality, formulated in a particular way:

MAXIM OF QUALITY (KNOWLEDGE NORM OF ASSERTION)

For any context $c, s_{c}$ must assert $\langle\phi\rangle^{c}$ in $c$ only if $s_{c}$ knows $\langle\phi\rangle^{c}$ in $c$ (Gazdar 1979; Williamson 1996).

Here $s_{c}$ is the speaker of $c$ and $\langle\phi\rangle^{c}$ is the proposition expressed by $\phi$ in $c$. So if a speaker asserts $\langle\phi\rangle^{c}$, this will normally imply that the speaker knows $\langle\phi\rangle^{c}$, since the speaker will normally be assumed to be attempting to satisfy the above maxim. The second claim is a (not uncontroversial) principle concerning the epistemology of taste:

\section{ACQUAINTANCE PRINCIPLE}

Normally, in an autocentric context $c, s_{c}$ knows whether $\langle a \text { is delicious }\rangle^{c}$ is true only if $s_{c}$ has tasted $a$ prior to $t_{c}$ in $w_{c}$ (Wollheim 1980; Ninan 2014). ${ }^{2}$

If we restrict ourselves to situations in which taste predicates are used autocentrically, this means that if the speaker knows that $a$ is delicious, that will tend to imply that the speaker has tasted $a$, and if the speaker knows that $a$ is not delicious, that will also tend to imply that the speaker has tasted $a$.

The epistemic view predicts the initial data that led us to the presupposition view:

(13) a. The carrot cake is delicious.

b. The carrot cake is not delicious-it isn't moist enough.

$\hookrightarrow$ the speaker has tasted the cake

If a speaker utters (13a), for example, this will imply, via QUALITY that she knows that the cake is delicious. This, in turn, will imply, via the ACQUAINTANCE PRINCIPLE, that she has tasted the cake. A similar account will apply to (13b).

According to the epistemic view, (14) is akin to the Moore-paradoxical (15):

(14) ? The carrot cake is delicious, but I haven't tasted it.

(15) ? Mary was at the office, but I don't know that Mary was at the office.

Both (14) and (15) will be bad for essentially the same reason. If you assert (14), for example, this will imply that you know that conjunction. Assuming that you know a

2 Here $a$ is assumed to be a rigid singular term of the object language, and $a$ is also used in the metalanguage to pick out the denotation of object language $a$. 
conjunction only if you know each conjunct, this will imply that you know that the cake is delicious. Given the ACQUAINTANCE PRINCIPLE, this will in turn imply that you have tasted the cake before, which is precisely what the second conjunct denies.

We noted earlier that data involving modals, conditionals, and questions posed a prima facie problem for the presupposition view. In contrast, the epistemic view seems to avoid this problem. For note that the ACQUAINTANCE PRINCIPLE only concerns atomic taste sentences and their negations. That means the epistemic view simply doesn't say anything about other kinds of embeddings. So, unlike the presupposition view, it doesn't predict that the acquaintance inference will project over modals, conditional operators, etc.. Furthermore, the acquaintance inference does seem to pattern like a Quality implicature here; compare (16) and (17):

a. The carrot cake must have been delicious.

b. The carrot cake might have been delicious.

c. If the carrot cake was delicious, Mary will be pleased.

d. Was the carrot cake delicious?

$\leftrightarrow$ the speaker has tasted the cake

(17) a. Mary must have been at the office. ${ }^{3}$

b. Mary might have been at the office.

c. If Mary was at the office, she'll be tired.

d. Was Mary at the office?

4 the speaker knows Mary was at the office

So the epistemic view has certain virtues (see Ninan 2014 for a more extensive discussion). Yet the epistemic view also faces certain challenges, as a number of authors have observed. ${ }^{4}$ A thorough assessment of the epistemic view would require us to consider each of these objections in detail, but in keeping with our focus on the projection profile of the acquaintance inference, I want to examine some problems that arise specifically in connection with projection data.

Let us proceed systematically, examining first how the acquaintance requirement interacts with the Boolean connectives (negation, conjunction, disjunction) and then how it interacts with generalized quantifiers.

Now we've already discussed the fact that the acquaintance inference projects over negation, and we also saw in passing that it projects over conjunction. At

3 Epistemic must poses a potential problem for the epistemic view. Given QUALITY, asserting must $\phi$ will imply $K$ (must $\phi$ ). If must $\phi$ entails $\phi$ (von Fintel \& Gillies 2010), then if knowledge is closed under entailment, this will imply $K \phi$. See Ninan (2014: 304-305) for discussion.

4 See Anand \& Korotkova 2018; Franzén 2018; Muñoz 2019; Dinges \& Zakkou 2020; Willer \& Kennedy Forthcoming. 
The Projection Problem for Predicates of Taste

least, that is one lesson we might draw from the infelicity of (14). The following less-distracting example seems to confirm this:

(18) The cake is delicious and it is gluten-free.

$\hookrightarrow$ the speaker has tasted the cake

But these facts about negation and conjunction do not pose a problem for the epistemic view. The negation datum is essentially built into the ACQUAINTANCE PRINCIPLE. If we use $T a$ to translate The cake is delicious, $K \phi$ to translate The speaker knows $\phi$, and $A a$ to translate The speaker has tasted the cake, then the ACQUAINTANCE PRINCIPLE can be formulated as: $(K T a \hookrightarrow A a) \wedge(K \neg T a \hookrightarrow A a)$. The conjunction data also falls out of the epistemic view given a fairly plausible principle concerning knowledge of conjunctions, a principle we appealed to above in our discussion of (14): $K(\phi \wedge \psi) \hookrightarrow(K \phi \wedge K \psi){ }^{5}$

But things are not so smooth when we turn to disjunction. Cariani (Forthcoming: $\S 13.8$ ) observes that a disjunction of simple taste sentences seems to give rise to a disjunction of acquaintance claims. For example:

$A$ has just arrived at the wedding banquet. He's hungry.

a. $[A]$ : What's good here?

b. $[B]$ : Either the moussaka is delicious or the lasagna is-I couldn't tell which was which.

$\hookrightarrow$ the speaker has tasted the moussaka or the speaker has tasted the lasagna

Note that it wouldn't be quite accurate to say the acquaintance requirement projects over disjunction-(19b) doesn't imply that the speaker has tasted the moussaka, for example-but it does seem to be interpreted. That the atomic sentence The moussaka is delicious comes with an acquaintance requirement seems to affect the overall interpretation of the complex sentence $(19 \mathrm{~b})$ in which that atomic sentence occurs.

This is a prima facie problem for the epistemic view. From the fact that the speaker utters a disjunction of atomic taste sentences $(T a \vee T b)$, QUALITY implies that the speaker knows that disjunction, $K(T a \vee T b)$. But now the epistemic view is stuck, for the ACQUAINTANCE PRINCIPLE simply says nothing about what $K(T a \vee$ $T b)$ implies about what the speaker has tasted. It would imply a disjunction of acquaintance claims if we could move from $K(T a \vee T b)$ to $(K T a \vee K T b)$, but such a move is not valid in general, nor is it plausible in the specific case at hand.

Quantifiers raise a similar problem. Take (20), for example:

5 We are using $\phi \hookrightarrow \psi$ to mean that $\phi$ either implicates or entails $\psi$. 
(20) Something on the dessert table is delicious.

$\hookrightarrow$ something on the dessert table is such that the speaker has tasted it

As indicated, this seems to imply that the speaker has tasted something on the dessert table. But, again, the epistemic view doesn't seem to predict this. By QUALITY, the speaker's uttering some $_{x}(F x)(T x)$ will imply $K\left(\operatorname{some}_{x}(F x)(T x)\right)$. But, again, the ACQUAINTANCE PRINCIPLE simply says nothing about what $K\left(\operatorname{some}_{x}(F x)(T x)\right)$ implies about what the speaker has tasted.

I suspect that there are some moves a defender of the epistemic view could make in response to these problems. But if we step back a bit, it seems that these data really do support the idea that the acquaintance requirement is a presupposition of some kind. This is because, with respect to Boolean connectives and existential quantification, the acquaintance requirement seems to be behaving quite like a presupposition. Consider:

(21) Mary stopped smoking and she feels much better now.

$\hookrightarrow$ Mary smoked in the past

(22) Mary stopped smoking or John stopped smoking.

$\hookrightarrow$ Mary smoked in the past or John smoked in the past

(23) Some student stopped smoking.

$\hookrightarrow$ some student smoked in the past

(Compare each $(n)$ here with its taste predicate counterpart $(n-3)$.) So even if these data do not refute the epistemic view, they do provide us with some motivation for examining the presupposition-plus-obviation view in more detail. But before we do that, it will be useful to reflect on some of the foregoing data and what they might suggest about which operators obviate the acquaintance requirement and which do not. For when we pay explicit attention to this question, a pattern begins to emerge.

\section{An apparent pattern}

Note first that the above discussion of negation, conjunction, and disjunction suggest that these operators do not obviate the acquaintance requirement. And sentence (20) suggests that the existential quantifier does not obviate it either, at least not when a taste predicate occurs in its nuclear scope. We find something similar with other quantifiers as well:

(24) Everything on the dessert table is tasty.

$\hookrightarrow$ everything on the dessert table is such that the speaker has tasted it

(25) Nothing on the dessert table is tasty.

$\hookrightarrow$ everything on the dessert table is such that the speaker has tasted it 
The Projection Problem for Predicates of Taste

As we shall see in $\S 4$, this pattern continues with other quantifiers as well.

If we say that an extensional operator is one that does not shift the world of evaluation, then it seems natural to suppose that Boolean connectives and generalized quantifiers are extensional operators. Thus, the foregoing data provide seems to provide some motivation for the following generalization:

\section{EXTENSIONAL CONJECTURE}

No extensional operator obviates the acquaintance inference.

Now recall the data that we cited as an initial problem for the presupposition view. That data consisted of epistemic modals, indicative conditionals, and questions. If we set the last of these aside, the generalization here seems to be that modals obviate the acquaintance inference, as Klecha (2014) and Cariani (Forthcoming: $\S 13.7)$ suggest. Future operators might be taken to further confirm this hypothesis, since as Klecha observes, future operators also obviate the acquaintance inference:

(26) The cookies in the oven will be tasty when they're done. It's a shame that they contain arsenic and so will have to be destroyed. (Ninan 2014: 305)

$\leftrightarrow$ the speaker has tasted/will taste the cookies

And on many views, future operators are themselves modal operators of some kind (e.g. Kaufmann 2005; Copley 2009; Cariani \& Santorio 2018).

Let's say an operator is intensional just in case it shifts the world of evaluation. Assuming modals are intensional operators in this sense, we might state our second conjecture as follows:

\section{INTENSIONAL CONJECTURE}

All intensional operators obviate the acquaintance inference.

This would seem to cover indicative conditionals as well, since on standard approaches, these constructions are analyzed as containing an intensional operator. Note that if we put our two conjectures together, we get:

\section{STRONG CONJECTURE}

An operator $O$ obviates the acquaintance inference iff $O$ is an intensional operator.

As its name suggest, this conjecture is quite strong, and so we should not be too surprised if there are exceptions to this rule. Still, it would be interesting if we had uncovered a general tendency here, even if it isn't an exceptionless law. And even if the STRONG CONJECTURE ultimately proves to be false, examining what it gets right and what it gets wrong may lead us toward a more adequate generalization concerning which operators obviate the acquaintance requirement and which do not. 


\section{The presupposition-plus-obviation view}

Let's consider how we might construct a version of the presupposition-plus-obviation view that captures the foregoing data. In particular, we seek a theory that accounts for how the acquaintance requirement interacts with Boolean connectives and generalized quantifiers, and that also allows intensional operators to obviate the acquaintance requirement. In constructing this theory, we are loosely guided by our STRONG CONJECTURE. We discuss the the relationship between our theory and the STRONG CONJECTURE in more detail in $\$ 5$, where we also consider some further evidence that bears on that conjecture.

As I noted earlier, the basic idea behind the presupposition-plus-obviation view can be found in Anand \& Korotkova 2018 and Willer \& Kennedy Forthcoming. Our account also builds in various way on other work on taste predicates (e.g. Lasersohn 2005; Stephenson 2007a,b; Sæbø 2009; MacFarlane 2014); on trivalent theories of presupposition projection (e.g. Peters 1979; Beaver \& Krahmer 2001; Fox 2008; George 2008a,b; Schlenker 2008); and on supervaluationism (Thomason 1970, 1984; MacFarlane 2014).

\subsection{Basic framework}

Let's start with the idea that, at a point of evaluation $e, a$ is delicious says that $a$ is delicious according to the standard of taste determined by $e$. That raises an obvious question: what is a standard of taste? Here is what MacFarlane has to say about this notion:

Talk of a standard can suggest something intellectual: a set of principles the agent uses in assessing whether something is tasty. Nothing like that is intended here. Think of a standard, rather, as something that determines a scale...

I... insist on an analytic connection between one's tastes and what flavors one likes. Roughly: if one knows a flavor and likes it, then that flavor is evaluated positively by one's tastes; if one dislikes it, then that flavor is evaluated negatively by one's tastes; and if one neither likes nor dislikes it, then the flavor is evaluated neutrally by one's tastes. I don't think we can make much sense of the idea that a person might have a taste for overripe peaches but not like them, or love the flavor of licorice but not have a taste for it. (MacFarlane 2014: 143-144)

We model a standard of taste as a (possibly partial) function from objects in a given domain to $\{0,1\}$, where $f(o)=1$ if $o$ is tasty according to $f$, and $f(o)=0$ if it is 
The Projection Problem for Predicates of Taste

not the case that $o$ is tasty according to $f$ (so $f(o)=0$ covers both the case where $o$ is neither good nor bad according to $f$ and the case where it is bad according to $f){ }^{6}$

An important question for us is whether an agent's standard of taste is defined for items that the agent has not (yet) tasted. But rather than answer this question, we can simply distinguish between two types of standards of taste, both of which can be modeled as functions from a background domain to $\{0,1\}$. Assume we have a (non-empty) set of worlds $W$ and a (non-empty) finite set of individuals $D$. Then we may distinguish between an agent's dispositional standard of taste from their categorical standard of taste as follows.

Definition 1. An agent $j$ 's dispositional standard of taste in world $w, \delta^{w, j}$, is a total function from $D$ to $\{0,1\}$.

In a more explicit model-theoretic development, we would take $\delta$ to be an element of the model (see below). And in the intended model, we assume that the following would hold:

- $\delta^{w, j}(o)=1$ if $j$ is disposed to like $o$ in $w$, and

- $\delta^{w, j}(o)=0$ if $j$ is disposed not to like $o$ in $w$.

As a rough rule-of-thumb, we may say that $j$ is is disposed (not) to like $o$ in $w$ just in case it is true in $w$ that if $j$ were to try $o$, she would (not) like it. ${ }^{7}$

Definition 2. An agent $j$ 's categorical standard of taste in world $w, \chi^{w, j}$, is a (possibly partial) function from $D$ to $\{0,1\}$ subject to the constraint that for any $o \in D$, if $o \in \operatorname{dom}\left(\chi^{w, j}\right)$, then $\chi^{w, j}(o)=\delta^{w, j}(o)$.

In the intended model, $\chi^{w, j}$ will simply be the restriction of $\delta^{w, j}$ to the things $j$ has tasted in $w$. So $\chi^{w, j}$ will typically be a partial function, since most of us have not tasted everything in the domain of discourse. Thus, in the intended model, we would have roughly the following:

- $\chi^{w, j}(o)=1$ if $j$ has tasted and liked $o$ in $w$,

- $\chi^{w, j}(o)=0$ if $j$ has tasted $o$ in $w$ and it is not the case that $j$ liked $o$ in $w$, and

6 Given that taste predicates are gradable adjectives, it might be better to think of a standard of taste as something that yields a more fine-grained ordering of items according to their tastiness, rather than as simply imposing a binary division on the domain into tasty and not-tasty (note MacFarlane's mention of a scale). While the theory presented in the text could be enriched in this way, the coarse-grained approach we take appears to suffice for our purposes here.

7 There is controversy as to whether dispositions can be analyzed in terms of counterfactuals (see e.g. Shope 1978; Bonevac, Dever \& Sosa 2006), but we don't intend to enter into this dispute-the above is offered only as a rough rule-of-thumb. 
- $o \notin \operatorname{dom}\left(\chi^{w, j}\right)$ if $j$ hasn't tasted $o$ in $w$.

We ignore the possibility that one is disposed to like something, but didn't like it on a particular occasion because of some extenuating circumstance (e.g. one was feeling ill). So if, for example, you have tried sea urchin, both your dispositional and your categorical standards will evaluate it in the same way. But if you have not tried sea urchin, your dispositional standard still evaluates it, while your categorical standard does not.

To state our semantics, it will help to adopt a few more definitions. Let's say that a centered world is a pair of a world and an individual (we ignore times throughout for simplicity).

Definition 3. A (taste) generator is a (total) function from centered worlds to standards of taste.

A generator takes a centered world and 'generates' a standard of taste. We assume that $\delta$ and $\chi$ are generators in this sense (where $\sigma$ is a generator, we write $\sigma^{w, j}$ for $\sigma(w, j))$.

Definition 4. A generator $\sigma$ is complete iff for all $(w, j), \sigma^{w, j}$ is a total function.

Definition 5. If $\sigma$ and $\sigma^{\prime}$ are both generators, then $\sigma^{\prime}$ is a complete extension of $\sigma$, $\sigma^{\prime} \succeq \sigma$, iff

i. $\sigma^{\prime}$ is complete, and

ii. for all $(w, j)$ and all $o \in D$, if $o \in \operatorname{dom}\left(\sigma^{w, j}\right)$, then $\sigma^{\prime w, j}(o)=\sigma^{w, j}(o)$.

So if $\sigma^{\prime}$ is a complete extension of $\sigma$, then $\sigma^{\prime w, j}$ agrees with $\sigma^{w, j}$ on all the cases that $\sigma^{w, j}$ decides, but then goes on and decides all other cases as well.

In what follows, we will mostly be interested in complete extensions of $\chi$, the 'categorical generator'. Note that $\delta$ is a complete extension of $\chi$, since $\delta$ is complete, and $\delta^{w, j}$ and $\chi^{w, j}$ agree on all the cases that $\chi^{w, j}$ decides. But there are other complete extensions of $\chi$ as well; two in particular stand out:

Definition 6. The 'picky' generator $\sigma_{0}$ defined as follows:

i. $\sigma_{0} \succeq \chi$, and

ii. for all $(w, j)$ and all $o \notin \operatorname{dom}\left(\chi^{w, j}\right), \sigma_{0}^{w, j}(o)=0$.

So $\sigma_{0}^{w, j}$ maps everything not in the domain of $\chi^{w, j}$ to $0 ; \sigma_{0}^{w, j}$ is picky in that it 'doesn't like' anything it hasn't tried. 
The Projection Problem for Predicates of Taste

Definition 7. The 'easy-to-please' generator $\sigma_{1}$ defined as follows:

i. $\sigma_{1} \succeq \chi$, and

ii. for all $(w, j)$ and all $o \notin \operatorname{dom}\left(\chi^{w, j}\right), \sigma_{1}^{w, j}(o)=1$.

So $\sigma_{1}^{w, j}$ maps everything not in the domain of $\chi^{w, j}$ to $1 ; \sigma_{0}^{w, j}$ is easy to please in that it 'likes' everything it hasn't tried. The main role these two generators play in what follows is technical: proofs of various facts about the system below can usually be found by adverting to one or both of these generators. ${ }^{8}$

We state the semantics for a first-order language whose vocabulary includes variables, individual constants, $n$-ary predicates (including a distinguished one-place taste predicate $T$ ), Boolean connectives, generalized quantifiers, epistemic modals, and attitude verbs. A model for the language would be an $n$-tuple that includes the following elements: $W, D, \delta, \chi$ (where these elements are characterized as above), and an interpretation function $I$, where $I$ assigns an element of $D$ to each individual constant, and a function from worlds to subsets of $D^{n}$ to all $n$-ary predicates other than the taste predicate $T$.

Where $t$ is a term (individual constant or variable), the denotation of $t, t^{g}$, is $g(t)$ if $t$ is a variable, and $I(t)$ otherwise. If $t$ is an individual constant, we write " $t$ " instead of " $I(t)$ ".

The semantics consists of two components: a recursive definition of truth at a point of evaluation, and then a ('postsemantic') definition of truth at a context (Kaplan 1989; MacFarlane 2014). A point of evaluation is an $n$-tuple $(w, j, \sigma, g)$ consisting a world $w$, a judge $j$, a complete generator $\sigma$, and a variable assignment $g .{ }^{9}$ Since the generator $\sigma$ in any point of evaluation is complete, truth at a point of evaluation is bivalent: for any point $e$ and any sentence $\phi, \phi$ is either true at $e$ or false at $e$. Truth-value gaps are introduced at the second step, in the definition of truth at a context.

The clauses for atomic sentences are as follows: ${ }^{10}$

(S1) $\llbracket P t_{1}, \ldots, t_{n} \rrbracket^{w, j, \sigma, g}=1$ iff $\left(t_{1}^{g}, \ldots, t_{n}^{g}\right) \in I(P)(w)$, where $P$ is any $n$-ary predicate

8 It is possible that we could restrict the set of complete extensions over which we supervaluate to just these two complete extensions of $\chi$; see George (2008b) for related discussion concerning presuppositions in the Strong Kleene setting.

9 Coppock (2018) argues for a semantics for taste predicates in which pairs of worlds and judges are replaced by a single parameter called an outlook, which (in her setup) is a refinement of a possible world. I suspect the present proposal could be integrated into that framework, but I leave the issue for future inquiry.

10 Statements (S1)-(S15) constitute the recursive definition of truth at a point of evaluation. The definition is not given all at once, but instead presented over the course of the remainder in order to facilitate discussion of individual clauses. 
other than $T$

$$
\llbracket T t \rrbracket^{w, j, \sigma, g}=1 \text { iff } \sigma^{w, j}\left(t^{g}\right)=1
$$

If we let $\mathrm{Ta}$ translate the cake is delicious, this yields the following truth-at-a-point conditions for $T a$ :

$$
\llbracket T a \rrbracket^{w, j, \sigma, g}=1 \text { iff } \sigma^{w, j}(a)=1
$$

A context $c$ is an $n$-tuple $\left(w_{c}, s_{c}, j_{c}, g_{c}\right)$ consisting of a world $w_{c}$, a speaker $s_{c}$, a judge $j_{c}$, and a variable assignment $g_{c}$. The reason for distinguishing between the speaker and the judge of the context is to allow for both autocentric and exocentric readings of taste predicates: when the judge is the speaker, we get an autocentric reading, when judge and speaker are distinct, we get an exocentric reading. We then define truth (falsity) at a context by supervaluating over the complete extensions of $\chi$ :

\section{SUPERVALUATIONIST TRUTH AT A CONTEXT}

Sentence $\phi$ is true at $c,[\phi]^{c}=1$, iff for all $\sigma \succeq \chi, \llbracket \phi \rrbracket^{w_{c}, j_{c}, \sigma, g_{c}}=1$.

Sentence $\phi$ is false at $c,[\phi]^{c}=0$ iff for all $\sigma \succeq \chi, \llbracket \phi \rrbracket^{w_{c}, j_{c}, \sigma, g_{c}}=0$.

This semantics predicts that if The cake delicious is true in an autocentric context, then the speaker will have tasted and liked the cake. For we have:

Fact 1. $[T a]^{c}=1$ only if $\chi^{w_{c}, j_{c}}(a)=1$.

Proof. Suppose $[\mathrm{Ta}]^{c}=1$. Then for all $\sigma \succeq \chi, \llbracket T a \rrbracket^{w_{c}, j_{c}, \sigma, g_{c}}=1$. So for all $\sigma \succeq \chi$, $\sigma^{w_{c}, j_{c}}(a)=1$. Suppose, for reductio $\chi^{w_{c}, j_{c}}(a) \neq 1$. Then either $\chi^{w_{c}, j_{c}}(a)=0$ or $a \notin \operatorname{dom}\left(\chi^{w_{c}, j_{c}}\right)$. In either case, the picky extension of $\chi, \sigma_{0}$, will be such that

$\sigma_{0}^{w_{c}, j_{c}}(a)=0$. But since $\sigma_{0} \succeq \chi$, that contradicts the claim that for all $\sigma \succeq \chi$, $\sigma^{w_{c}, j_{c}}(a)=1$.

\subsection{Extensional operators}

We adopt the standard classical recursive clauses for the Boolean connectives:

$$
\begin{aligned}
& \llbracket \neg \phi \rrbracket^{w, j, \sigma, g}=1 \text { iff } \llbracket \phi \rrbracket^{w, j, \sigma, g}=0 \\
& \llbracket \phi \wedge \psi \rrbracket^{w, j, \sigma, g}=1 \text { iff } \llbracket \phi \rrbracket^{w, j, \sigma, g}=\llbracket \phi \rrbracket^{w, j, \sigma, g}=1 \\
& \llbracket \phi \vee \psi \rrbracket^{w, j, \sigma, g}=1 \text { iff } \llbracket \phi \rrbracket^{w, j, \sigma, g}=1 \text { or } \llbracket \psi \rrbracket^{w, j, \sigma, g}=1
\end{aligned}
$$


The Projection Problem for Predicates of Taste

When paired with our definition of truth at a context, we predict our earlier observations concerning these connectives. For example, if you say, The cake is not delicious, this will imply that you tasted and didn't like the cake:

Fact 2. $[\neg T a]^{c}=1$ only if $\chi^{w_{c}, j_{c}}(a)=0$.

(Proofs of Facts 2-4 are left to the reader.) And if you say The cake is delicious and it's gluten-free, this will imply that you tasted and liked the cake:

Fact 3. $[\text { Ta } \wedge \phi]^{c}=1$ only if $\chi^{w_{c}, j_{c}}(a)=1$.

And if you say, Either the cake is delicious or the pie is, this will imply that either you tasted and liked the cake or you tasted and liked the pie:

Fact 4. $[T a \vee T b]^{c}=1$ only if $\chi^{w_{c}, j_{c}}(a)=1$ or $\chi^{w_{c}, j_{c}}(b)=1$.

Note that this implies Cariani's observation-the disjunction implies that you tasted at least one of them-but it in fact implies something stronger: that you tasted and liked at least one of them. So if, for example, you tasted the pie and didn't like it, and you didn't taste the cake but are disposed to like it, the disjunction will not be true at your context (we restrict ourselves to autocentric contexts here and in what follows). ${ }^{11}$

Our recursive clauses for generalized quantifiers are again the standard ones:

$$
\begin{aligned}
\text { (S6) } & \llbracket \operatorname{some}_{x}(\phi)(\psi) \rrbracket^{w, j, \sigma, g}=1 \text { iff } \\
& \left\{o \in D: \llbracket \phi \rrbracket^{w, j, \sigma, g[x / o]}=1\right\} \cap\left\{o \in D: \llbracket \psi \rrbracket^{w, j, \sigma, g[x / o]}=1\right\} \neq \emptyset^{12} \\
\text { (S7) } & \llbracket \text { every }_{x}(\phi)(\psi) \rrbracket^{w, j, \sigma, g}=1 \text { iff } \\
& \left\{o \in D: \llbracket \phi \rrbracket^{w, j, \sigma, g[x / o]}=1\right\} \subseteq\left\{o \in D: \llbracket \psi \rrbracket^{w, j, \sigma, g[x / o]}=1\right\}
\end{aligned}
$$

$$
\begin{aligned}
& \llbracket n o_{x}(\phi)(\psi) \rrbracket^{w, j, \sigma, g}=1 \text { iff } \\
& \left\{o \in D: \llbracket \phi \rrbracket^{w, j, \sigma, g[x / o]}=1\right\} \cap\left\{o \in D: \llbracket \psi \rrbracket^{w, j, \sigma, g[x / o]}=1\right\}=\emptyset \\
& \llbracket \operatorname{most}_{x}(\phi)(\psi) \rrbracket^{w, j, \sigma, g}=1 \text { iff } \\
& \left|\left\{o \in D: \llbracket \phi \rrbracket^{w, j, \sigma, g[x / o]}=1\right\} \cap\left\{o \in D: \llbracket \psi \rrbracket^{w, j, \sigma, g[x / o]}=1\right\}\right|> \\
& \left|\left\{o \in D: \llbracket \phi \rrbracket^{w, j, \sigma, g[x / o]}=1\right\}-\left\{o \in D: \llbracket \psi \rrbracket^{w, j, \sigma, g[x / o]}=1\right\}\right|
\end{aligned}
$$

$$
\begin{aligned}
& \llbracket \text { exactly two } x(\phi)(\psi) \rrbracket^{w, j, \sigma, g}=1 \text { iff } \\
& \left|\left\{o \in D: \llbracket \phi \rrbracket^{w, j, \sigma, g[x / o]}=1\right\} \cap\left\{o \in D: \llbracket \psi \rrbracket^{w, j, \sigma, g[x / o]}=1\right\}\right|=2
\end{aligned}
$$

11 A further point about disjunction is worth noting: $T a \vee \neg T a$ is true at every context. This means that Either the cake is delicious or it isn't will not imply that one has tasted the cake; this appears to be a welcome result (Cariani Forthcoming: $\$ 13.8$ ).

12 For any variable assignment $g, g[x / o]$ is the assignment $h$ such that $h(x)=o$ and is otherwise like $g$. 
(S11) «at least two $o_{x}(\phi, \psi) \rrbracket^{w, j, \sigma, g}=1$ iff

$$
\left|\left\{o \in D: \llbracket \phi \rrbracket^{w, j, \sigma, g[x / o]}=1\right\} \cap\left\{o \in D: \llbracket \psi \rrbracket^{w, j, \sigma, g[x / o]}=1\right\}\right| \geq 2
$$

(S12) $\llbracket$ at most two $(\phi, \psi) \rrbracket^{w, j, \sigma, g}=1$ iff

$$
\left|\left\{o \in D: \llbracket \phi \rrbracket^{w, j, \sigma, g[x / o]}=1\right\} \cap\left\{o \in D: \llbracket \psi \rrbracket^{w, j, \sigma, g[x / o]}=1\right\}\right| \leq 2
$$

This yields a number of results of interest. We can start with a general result that pertains to all generalized quantifiers. To state it, first note that for each generalized quantifier $Q_{x}$, there is a corresponding binary relation $Q_{R}$ on subsets $A, B$ of $D$ such that:

$$
\begin{aligned}
& \llbracket Q_{x}(\phi, \psi) \rrbracket^{w, j, \sigma, g}=1 \text { iff } \\
& Q_{R}\left(\left\{o \in D: \llbracket \phi \rrbracket^{w, j, \sigma, g[x / o]}=1\right\},\left\{o \in D: \llbracket \psi \rrbracket^{w, j, \sigma, g[x / o]}=1\right\}\right)
\end{aligned}
$$

For example:

$$
\begin{array}{llll}
\text { some }_{R}: & A \cap B \neq \emptyset & \text { most }_{R}: & |A \cap B|>|A-B| \\
\text { every }_{R}: & A \subseteq B & \text { exactly two } & R
\end{array}
$$

Then we have:

Fact 5. For any generalized quantifier $Q_{x}$ and corresponding binary relation $Q_{R}$ on subsets of D: if $\left[Q_{x}(F x, T x)\right]^{c}=1$, then $Q_{R}\left(I(F)\left(w_{c}\right),\left\{o: \chi^{w_{c}, j_{c}}(o)=1\right\}\right)$.

Proof. Suppose $\left[Q_{x}(F x, T x)\right]^{c}=1$. So for all $\sigma \succeq \chi, \llbracket Q_{x}(F x, T x) \rrbracket^{w_{c}, j_{c}, \sigma, g_{c}}=1$. So for all $\sigma \succeq \chi, Q_{R}\left(I(F)\left(w_{c}\right),\left\{o \in D: \sigma^{w_{c}, j_{c}}(o)=1\right\}\right)$. So where $\sigma_{0}$ is the picky extension of $\chi$, we have $Q_{R}\left(I(F)\left(w_{c}\right),\left\{o \in D: \sigma_{0}^{w_{c}, j_{c}}(o)=1\right\}\right)$, since $\sigma_{0} \succeq \chi$. And note that we have the following equivalence:

$$
\left\{o \in D: \chi^{w_{c}, j_{c}}(o)=1\right\}=\left\{o \in D: \sigma_{0}^{w_{c}, j_{c}}(o)=1\right\} .
$$

Thus, $Q_{R}\left(I(F)\left(w_{c}\right),\left\{o \in D: \chi^{w_{c}, j_{c}}(o)=1\right\}\right)$, which is what we needed to show.

So if you say $Q$ things on the dessert table are delicious, this will imply that $Q$ things on the dessert table are such that you tasted and liked them. For example, if you say, Something on the dessert table is delicious, this will imply that there is something on the dessert table that you tasted and liked. Note again that this is stronger than just: there is something on the dessert table that you tasted. It's not enough that you have tasted something on the table, didn't like it, but are disposed to like something else on the table that you didn't try. Similarly, if you say, Exactly two things on the dessert table are delicious, this implies that exactly two things on the table are such that you tasted and liked them. 
The Projection Problem for Predicates of Taste

There are some more specific results pertaining to particular quantifiers. Some of these are of interest because they reveal some subtle differences between the acquaintance requirement and standard presuppositions. For example, we have:

Fact 6. If $\left[\text { exactly two } o_{x}(F x, T x)\right]^{c}=1$, then $I(F)\left(w_{c}\right) \subseteq \operatorname{dom}\left(\chi^{w_{c}, j_{c}}\right)$.

Proof. Suppose $\left[\text { EXACTLY } \operatorname{TWO}_{x}(F x, T x)\right]^{c}=1$. So for all $\sigma \succeq \chi$ :

$$
\left|I(F)\left(w_{c}\right) \cap\left\{o \in D: \sigma^{w_{c}, j_{c}}(o)=1\right\}\right|=2 .
$$

And note that, by Fact 5, we also have:

$$
\left|I(F)\left(w_{c}\right) \cap\left\{o \in D: \chi^{w_{c}, j_{c}}(o)=1\right\}\right|=2 .
$$

So let $o_{1}, o_{2}$ be distinct elements of $D$ such that:

$$
I(F)\left(w_{c}\right) \cap\left\{o \in D: \chi^{w_{c}, j_{c}}(o)=1\right\}=\left\{o_{1}, o_{2}\right\} .
$$

Now suppose, for reductio, that there is an $o \in I(F)\left(w_{c}\right)$ such that $o \notin \operatorname{dom}\left(\chi^{w_{c}, j_{c}}\right)$. Let $o_{3}$ be such an $o$. Note that $o_{3}$ is distinct from both $o_{1}$ and $o_{2}$ since the latter are both in $\operatorname{dom}\left(\chi^{w_{c}, j_{c}}\right)$ while $o_{3}$ is not. Note that the easy-to-please extension $\sigma_{1}$ of $\chi$ will be such that $\sigma_{1}^{w_{c}, j_{c}}\left(o_{1}\right)=\sigma_{1}^{w_{c}, j_{c}}\left(o_{2}\right)=\sigma_{1}^{w_{c}, j_{c}}\left(o_{3}\right)=1$. Thus:

$$
I(F)\left(w_{c}\right) \cap\left\{o \in D: \sigma_{1}^{w_{c}, j_{c}}(o)=1\right\}=\left\{o_{1}, o_{2}, o_{3}\right\} .
$$

But then:

$$
\left|I(F)\left(w_{c}\right) \cap\left\{o \in D: \sigma_{1}^{w_{c}, j_{c}}(o)=1\right\}\right|=3 .
$$

But since $\sigma_{1} \succeq \chi$, this contradicts the claim that for all $\sigma \succeq \chi$ :

$$
\left|I(F)\left(w_{c}\right) \cap\left\{o \in D: \sigma^{w_{c}, j_{c}}(o)=1\right\}\right|=2 .
$$

So if you say, Exactly two things on the dessert table are delicious, this implies that you've tasted everything on the dessert table. Note that our earlier result was that if you say, Exactly two things on the dessert table are delicious, this implies that you've tasted and liked exactly two things on the table. Together, the two results imply that if you say, Exactly two things on the dessert table are delicious, this will imply that you tasted everything on the dessert table, but only liked two of them.

One thing interesting about this result is that there's apparently an empirical difference here between the acquaintance requirement and standard presuppositions. Consider: 
(27) Exactly two students in my class stopped smoking recently.

Suppose there are ten students in my class, two of whom smoked in the past and no longer smoke, eight of whom never smoked. According to George (2008a: 13), (27) has a reading on which it is true in this situation. This consequence is a problem for the supervaluational treatment of presupposition triggers like stops (and for the Strong Kleene approach George develops), but the corresponding prediction seems right in the case of taste predicates.

A related result concerns at most two:

Fact 7. Let $\left|I(F)\left(w_{c}\right)\right|=n$, for some non-negative integer $n$. Then:

i. If $n>2$ and $[\text { at most two } x(F x)(T x)]^{c}=1$, then

$$
\left|I(F)\left(w_{c}\right) \cap\left\{o \in D: \chi^{w_{c}, j_{c}}(o)=0\right\}\right| \geq(n-2) .
$$

ii. If $n \leq 2$, then $[\text { at most two } x(F x)(T x)]^{c}=1$.

Proof. Part (i): Suppose $n>2$ and $\left[\text { at most two } o_{x}(F x)(T x)\right]^{c}=1$. From the latter, it follows that for all $\sigma \succeq \chi,\left|I(F)\left(w_{c}\right) \cap\left\{o \in D: \sigma^{w_{c}, j_{c}}(o)=1\right\}\right| \leq 2$. Suppose for reductio that:

$$
\left|I(F)\left(w_{c}\right) \cap\left\{o \in D: \chi^{w_{c}, j_{c}}(o)=0\right\}\right|<(n-2) .
$$

So:

$$
\left|I(F)\left(w_{c}\right) \cap\left\{o \in D: \chi^{w_{c}, j_{c}}(o)=0\right\}\right| \leq(n-3) .
$$

Since $\left|I(F)\left(w_{c}\right)\right|=n$, it must be that:

$$
\left|I(F)\left(w_{c}\right) \cap\left\{o \in D: \chi^{w_{c}, j_{c}}(o) \neq 0\right\}\right| \geq 3 .
$$

So let $o_{1}, o_{2}$, and $o_{3}$ be three distinct elements of $I(F)\left(w_{c}\right) \cap\left\{o \in D: \chi^{w_{c}, j_{c}}(o) \neq 0\right\}$. Note that our easy-to-please extension of $\chi, \sigma_{1}^{w_{c}, j_{c}}$, will map all three of these elements to 1 . Thus:

$$
\left|I(F)\left(w_{c}\right) \cap\left\{o \in D: \sigma_{1}^{w_{c}, j_{c}}(o)=1\right\}\right| \geq 3 .
$$

But since $\sigma_{1} \succeq \chi$, this contradicts the claim that for all $\sigma \succeq \chi$,

$$
\left|I(F)\left(w_{c}\right) \cap\left\{o \in D: \sigma^{w_{c}, j_{c}}(o)=1\right\}\right| \leq 2 .
$$

Part (ii): We are supposing that $\left|I(F)\left(w_{c}\right)\right|=n$, and that $n \leq 2$. Thus, every subset $A$ of $I(F)\left(w_{c}\right)$ is such that $|A| \leq 2$. Thus, for all $\sigma \succeq \chi$,

$$
\left|I(F)\left(w_{c}\right) \cap\left\{o \in D: \sigma^{w_{c}, j_{c}}(o)=1\right\}\right| \leq 2 .
$$


The Projection Problem for Predicates of Taste

And this suffices for the claim that $\left[\text { at most two } o_{x}(F x)(T x)\right]^{c}=1$.

To appreciate what claim (i) is saying, suppose there are ten things on the dessert table $(n=10)$. Then if At most two things on the dessert table are delicious is true in an autocentric context, this implies that the speaker has tasted and failed to like at least eight of them $(n-2=8)$. This result seems plausible to me, but, again, the corresponding result for standard presuppositions is less clear. If there are ten students in the class, and I say, At most two students quit smoking, does this imply that at least eight students smoked in the past and continue to smoke? That is not obvious. $^{13}$

Claim (ii) is a much more general fact about at most two $o_{x}$ and doesn't turn on anything specific to our treatment of taste predicates. I mention it here because it shows that the theory predicts that if there are at most two things on the dessert table, one can utter At most two things on the dessert table are delicious even if one has not tasted anything on the dessert table. ${ }^{14}$

\subsection{Intensional operators}

There is perhaps more to say about how the acquaintance requirement interacts with extensional operators, but we turn now to consider its interaction with intensional operators. Note that in the present system, an operator will obviate the acquaintance requirement if it shifts the generator parameter $\sigma$ to $\delta$, viz. the generator that maps each $(w, j)$ to $j$ 's dispositional standard at $w$ (cf. Anand \& Korotkova 2018). To see how this works, consider the following entry for must:

$$
\llbracket m u s t \phi \rrbracket^{w, j, \sigma, g}=1 \text { iff for all } w^{\prime} \in R(w), \llbracket \phi \rrbracket^{w^{\prime}, j, \delta, g}=1
$$

where $w^{\prime} \in R(w)$ iff $w^{\prime}$ is compatible with what is known in $w$. Note that in addition to shifting the world of evaluation, must shifts the generator parameter $\sigma$ to $\delta$. Together with our definition of truth at a context, this yields the following result:

Fact 8. $[\text { must Ta }]^{c}=1$ iff for all $w^{\prime} \in R\left(w_{c}\right): \delta^{w^{\prime}, j_{c}}(a)=1$.

(Proofs of Facts 8-10 are left to the reader.) Note that this condition does not require that $a$ is in $\operatorname{dom}\left(\chi^{w_{c}, j_{c}}\right)$, and so it can hold even if the judge/speaker has not tasted the cake.

Note that, on this account, if you say, The cake must be delicious in an autocentric context, you are saying that it follows from what is known that you are disposed to

13 For more discussion of quantifiers and presuppositions, see (in addition to the works cited earlier) Chemla 2009 and Fox 2012.

14 Note that if we defined exactly ${ }^{2} w o_{x}(\phi)(\psi)$ as (at least two $\left.x(\phi)(\psi)\right) \wedge\left(\right.$ at most two $\left.o_{x}(\phi)(\psi)\right)$, then Fact 6 would be a corollary of Facts 5 and 7.i. 
like the cake. The infelicity of the following may constitute modest evidence for this claim:

? The cake must be delicious, but I wouldn't like it if I tried it.

Perhaps I could say this if, for example, I would ordinarily like the cake, but wouldn't like it just now because I'm feeling ill. But otherwise, it seems that (28) would be odd.

We noted earlier that epistemic might and the indicative conditional also obviate the acquainted inference. We could predict these results by positing lexical entries for these operators according to which they shift the generator parameter $\sigma$ to $\delta$ (in addition to shifting the world parameter, as is standard). Thus, this semantic theory seems more or less adequate for handling most of the data discussed so far. It predicts that Boolean connectives and generalized quantifiers do not obviate the acquaintance requirement, and makes some interesting fine-grained predictions concerning the quantified cases. And it has a mechanism that allows intensional operators to obviate the acquaintance requirement; they can do this by shifting the generator parameter $\sigma$ to the dispositional generator $\delta$.

\section{The STRONG CONJECTURE revisited}

Recall our:

\section{STRONG CONJECTURE}

An operator $O$ obviates the acquaintance inference iff $O$ is an intensional operator.

In $\S 3$, we noted some evidence in favor of this conjecture, and we have yet to encounter any evidence against it. What is the status of this conjecture within the present theory?

Although our theory is compatible with this generalization, it doesn't entail it: our basic framework enables us to define operators that violate it. To see this, note that if we assume that an operator shifts the generator parameter iff it shifts it to $\delta$, then in our present theoretical setup, the STRONG CONJECTURE is equivalent to the following claim:

( ) An operator $O$ shifts the generator parameter iff $O$ shifts the world parameter.

Here is an example of a possible operator that violates $(\star)$ :

$$
\llbracket \text { shmust } \phi \rrbracket^{w, j, \sigma, g}=1 \text { iff for all } w^{\prime} \in R(w), \llbracket \phi \rrbracket^{w^{\prime}, j, \sigma, g}=1 .
$$


The Projection Problem for Predicates of Taste

This violates $(\star)$ in the right-to-left direction, shifting the world parameter without shifting the generator parameter. It's possible to define operators that violate $(\star)$ in the other direction as well.

But we could add $(\star)$ to our theory by simply stipulating that it is to count as a general rule of the grammar, one that constrains the possible lexical entries that any operator can have. Here are some examples of (unary) operators that would be permitted/proscribed by this rule:

$$
\begin{array}{rlrl}
\mathcal{\llbracket} \llbracket \phi \phi \rrbracket^{w, j, \sigma, g} & =1 \text { iff } \ldots \llbracket \phi \rrbracket^{w, j, \sigma, g^{\prime}} \ldots & \boldsymbol{X} \llbracket O \phi \rrbracket^{w, j, \sigma, g}=1 \text { iff } \ldots \llbracket \phi \rrbracket^{w^{\prime}, j, \sigma, g} \ldots \\
\mathcal{L} \llbracket O \phi \rrbracket^{w, j, \sigma, g}=1 \text { iff } \ldots \llbracket \phi \rrbracket^{w^{\prime}, j, \delta, g} \ldots & \boldsymbol{X} \llbracket O \phi \rrbracket^{w, j, \sigma, g}=1 \text { iff } \ldots \llbracket \phi \rrbracket^{w, j, \delta, g} \ldots
\end{array}
$$

But should we do this? Is ( $\star$ ) a rule of the grammar? The data we've examined so far would seem to support an affirmative answer to this question. But I want to close our discussion by considering some further evidence that bears on this question. We'll first look at some evidence from attitude verbs, evidence that arguably provides further support for $(\star)$. We'll then examine a potential problem for $(\star)$, one that arises in connection with disjunction.

\subsection{Attitude verbs}

The literature on taste predicates and attitude verbs generally seems to agree a sentence of the form $a$ believes/knows $b$ is tasty does not imply that the speaker has tasted $b$ nor does it imply that $a$ has tasted $b .{ }^{15}$ Consider believes, for example:

(29) John believes the carrot cake is delicious.

$\leftrightarrow$ the speaker has tasted the carrot cake

$\leftrightarrow$ John has tasted the carrot cake

This result follows from $(\star)$ together with the claim that believes shifts the world parameter.

To formulate a semantics for believes, we follow Stephenson (2007b: 63) in assuming that believes also shifts the judge parameter, so that the standard that matters for the the interpretation of the taste predicate is the one belonging to the bearer of the attitude, not the one belonging to the speaker.

$$
\llbracket B_{a} \phi \rrbracket^{w, j, \sigma, g}=1 \text { iff for all } w^{\prime} \in \mathrm{BEL}_{w, a}, \llbracket \phi \rrbracket^{w^{\prime}, a, \delta, g}=1
$$

15 See Stephenson (2007b: §2.5.2), Muñoz (2019), and Willer \& Kennedy (Forthcoming). As Muñoz: 64 and Willer \& Kennedy observe, the fact that knows obviates the acquaintance inference seems to conflict with the epistemic view's ACQUAINTANCE PRINCIPLE. Muñoz (2019: 165-169) bolsters the point with some interesting data concerning the interaction between taste predicates and Tibetan indirect evidentials. 
Here, $\mathrm{BEL}_{w, a}$ is the set of worlds compatible with what $a$ believes in $w$. Note that the operator $B_{a}$ is assumed to do three things: it shifts the world parameter, it shifts the judge parameter to $a$, and it shifts the generator parameter to $\delta$. This entry yields the following result:

Fact 9. $\llbracket B_{a} T b \rrbracket^{c}=1$ iff for all $w \in \mathrm{BEL}_{w_{c}, a}, \delta^{w, a}(b)=1$.

So if you say, John believes the carrot cake is delicious, you're saying that John believes that he is disposed to like the cake. And this, of course, doesn't imply that either the speaker or John has actually tasted the cake.

The attitude verb finds is an interesting case for us. Unlike the corresponding belief- and knowledge-ascriptions, a sentence of the form $a$ finds $b$ tasty does seem to imply that $a$ has tasted $b$ (Stephenson 2007b: 61):

(30) John finds the cake tasty.

$\hookrightarrow$ John has tasted the cake

So if finds were an intensional operator, it would be a counterexample to ( $\star$ ). Butrather interestingly from out point of view-according to Sæbø's (2009) influential treatment of finds, that verb is not an intensional operator in our sense. For on Sæbø's approach, finds does not shift the world of evaluation-it merely shifts the judge parameter. So $(\star)$ would require that it leave the generator parameter alone. ${ }^{16}$

Within the present framework, Sæbø’s idea would be implemented as follows:

$$
\llbracket F_{a} \phi \rrbracket^{w, j, \sigma, g}=1 \text { iff } \llbracket \phi \rrbracket^{w, a, \sigma, g}=1
$$

This yields the following result:

Fact 10. $\left[F_{a} T b\right]^{c}=1$ iff $\chi^{w_{c}, a}(b)=1$.

Thus, on this approach, John finds the cake tasty essentially says that John has tasted and liked the cake.

Thus, the foregoing data seem to provide some further support for taking $(\star)$ to be rule of the grammar. We now turn to an observation that seems to go in the other direction.

\subsection{Disjunction revisited}

Consider the following example:

(31) We're in a restaurant, about to order, and we see everyone around us eating lobster rolls. I say:

Either the lobster rolls here are delicious or they're out of everything else.

16 Though see Kennedy \& Willer (2016) for an alternative approach to finds. 
If the context is autocentric, then our theory predicts that the disjunction in (31) is true only if either (I've tasted and liked the lobster rolls) or (they're out of everything but lobster rolls). But that doesn't look correct. I can say (31) even if I know that I haven't tasted the lobster rolls in question. How should we respond to this problem?

There are at least two possible responses, one of which is consistent with the idea that $(\star)$ is a rule of the grammar, one of which is not. Let's start with the former.

According to the first line of response, what's going in (31) is that the taste predicate in (31) is being read exocentrically. Perhaps the relevant judge is actually a group, namely the group of patrons around us eating lobster rolls. In that case, our theory would predict that (31) is true in the relevant context only if either (the patrons have tasted and liked the lobster rolls) or (they're out of everything but lobster rolls). If this is what's going on, then we wouldn't seem to have a problem for our theory, nor would we have a counterexample to our putative rule $(\star)$. While this hypothesis strikes me as not wholly implausible, I must admit that it is not obvious that this is what's going on in this case.

A second response is to say that disjunction can (sometimes?) obviate the acquaintance requirement. One way to implement this idea is to add to our formal language a second disjunction-like operator $\nabla$ governed by the following semantic clause:

$$
\llbracket \phi \nabla \psi \rrbracket^{w, j, \sigma, g}=1 \text { iff } \llbracket \phi \rrbracket^{w, j, \delta, g}=1 \text { or } \llbracket \psi \rrbracket^{w, j, \delta, g}=1
$$

Note that $\nabla$ shifts the generator parameter $\sigma$ to $\delta$ (for both disjuncts), but doesn't shift the world parameter $w$. We could then say that natural language $o r$ is ambiguous between the non-obviating $\vee$ defined in (S4) and the obviating $\nabla$ defined above. If the disjunction in (31) is given the $\nabla$-reading, then if the context is autocentric, (31) will be true only if either (I'm disposed to like the lobster rolls) or (they're out of everything but lobster rolls). It's again not obvious that that's what that sentence is saying (in the relevant context), but at least this avoids the initial problem.

There may be a more elegant solution available along these lines-it would be nice to avoid having to say that or is ambiguous - but either way we are likely to have to reject the left-to-right direction of $(\star)$. For any solution along these lines would likely end up positing an extensional operator that shifts the generator parameter to $\delta .{ }^{17}$ But note that even if $(\star)$ fails in the left-to-right direction, it might still hold in the right-to-left direction, i.e. it might remain true that every intensional operator shifts the generator parameter and so obviates the acquaintance inference. We have yet to encounter a counterexample to that direction of $(\star)$, but, then again, we have yet to consider very many intensional operators. Other intensional operators to consider include deontic and other modals; counterfactual conditionals; and other attitude

17 Though see Zimmermann (2000) on the possibility that disjunction is an intensional operator. 
verbs. We leave these as a topics for future inquiry. Our STRONG CONJECTURE remains a mere conjecture.

\section{Summary}

We began by observing that the unusual projection profile of the acquaintance requirement makes its empirical status something of a puzzle. While it behaves quite like a presupposition with respect to some operators, it seems to disappear in the scope of certain standard 'presupposition holes.' But a more detailed examination of the projection data reveals a method in this madness, for it seems that while the acquaintance requirement behaves more-or-less like a presupposition with respect to extensional operators, it tends to be obviated by intensional operators. This pattern is nicely accommodated by the version of the presupposition-plus-obviation view that we developed above, but poses a serious challenge to the epistemic view discussed in Ninan (2014). That, at any rate, is the conclusion of the present inquiry.

\section{References}

Anand, Pranav \& Natasha Korotkova. 2018. Acquaintance content and obviation. Sinn und Bedeutung 22. 55-72.

Beaver, David \& Emiel Krahmer. 2001. A partial account of presupposition projection. Journal of Logic, Language, and Information 10(2). 147.

Bonevac, Daniel, Josh Dever \& David Sosa. 2006. The conditional fallacy. Philosophical Review 115(3). 273-316.

Bylinina, Lisa. 2017. Judge-dependence in degree constructions. Journal of Semantics 34(2). 291-331.

Cariani, Fabrizio. Forthcoming. The modal future: A theory of future-directed thought and talk. Cambridge: Cambridge University Press.

Cariani, Fabrizio \& Paolo Santorio. 2018. Will done better: Selection semantics, future credence, and indeterminacy. Mind 127(505). 129-165.

Chemla, Emmanuel. 2009. Presuppositions of quantified sentences: Experimental data. Natural Language Semantics 17(4). 299-340.

Copley, Bridget. 2009. The semantics of the future. Routledge.

Coppock, Elizabeth. 2018. Outlook-based semantics. Linguistics and Philosophy 41(2). 125-164.

Dinges, Alexander \& Julia Zakkou. 2020. Taste, traits, and tendencies. Philosophical Studies 1-24. Advance online publication.

von Fintel, Kai \& Anthony Gillies. 2010. Must... stay... strong! Natural Language Semantics 18(4). 351-383. 
The Projection Problem for Predicates of Taste

Fox, Danny. 2008. Two short notes on Schlenker's theory of presupposition projection. Theoretical linguistics 34(3). 237-252.

Fox, Danny. 2012. Presupposition projection from quantificational sentences: Trivalence, local accommodation, and presupposition strengthening. Unpublished manuscript, MIT.

Franzén, Nils. 2018. Aesthetic evaluation and first-hand experience. Australasian Journal of Philosophy 96(4). 669-682.

Gazdar, Gerald. 1979. Pragmatics. Academic Press.

George, B.R. 2008a. Predicting presupposition projection: Some alternatives in the strong Kleene tradition. Unpublished manuscript, UCLA.

George, B.R. 2008b. Presupposition repairs: A static, trivalent approach to predicting projection: UCLA MA thesis.

Kaplan, David. 1989. Demonstratives. In Joseph Almog, John Perry \& Howard Wettstein (eds.), Themes from Kaplan, 481-563. New York: Oxford University Press.

Kaufmann, Stefan. 2005. Conditional truth and future reference. Journal of Semantics 22(3). 231-280.

Kennedy, Christopher \& Malte Willer. 2016. Subjective attitudes and counterstance contingency. Semantics and Linguistic Theory 26. 913-933.

Klecha, Peter. 2014. Diagnosing modality in predictive expressions. Journal of Semantics 31(3). 443-455.

Lasersohn, Peter. 2005. Context dependence, disagreement, and predicates of personal taste. Linguistics and Philosophy 28(6). 643-686.

MacFarlane, John. 2014. Assessment-sensitivity: Relative truth and its applications. Oxford: Oxford University Press.

Mothersill, Mary. 1984. Beauty restored. Oxford: Oxford University Press.

Muñoz, Patrick. 2019. On tongues: The grammar of experiential evaluation: University of Chicago dissertation.

Ninan, Dilip. 2014. Taste predicates and the acquaintance inference. Semantics and Linguistic Theory 24. 290-309.

Pearson, Hazel. 2013. A judge-free semantics for predicates of personal taste. Journal of Semantics 30(3). 103-154.

Peters, Stanley. 1979. A truth-conditional formulation of Karttunen's account of presupposition. Synthese 40(2). 301-316.

Robson, Jon. 2012. Aesthetic testimony. Philosophy Compass 7(1). 1-10.

Sæbø, Kjell Johan. 2009. Judgment ascriptions. Linguistics and Philosophy 32(4). 327-352.

Schlenker, Philippe. 2008. Local contexts: Presuppositions, dynamic semantics and transparency. Unpublished early version of Schlenker 2009.

Schlenker, Philippe. 2009. Local contexts. Semantics and Pragmatics 2(3). 1-78. 
Shope, Robert K. 1978. The conditional fallacy in contemporary philosophy. The Journal of Philosophy 75(8). 397-413.

Stephenson, Tamina. 2007a. Judge dependence, epistemic modals, and predicates of personal taste. Linguistics and Philosophy 30(4). 487-525.

Stephenson, Tamina. 2007b. Towards a theory of subjective meaning: MIT dissertation.

Thomason, Richmond H. 1970. Indeterminist time and truth-value gaps. Theoria 36(3). 264-281.

Thomason, Richmond H. 1984. Combinations of tense and modality. In Handbook of philosophical logic, 135-165. Dordrecht: Springer.

Willer, Malte \& Chris Kennedy. Forthcoming. Assertion, expressions, experience. Inquiry.

Williamson, Timothy. 1996. Knowing and asserting. Philosophical Review 105(4). 489-523.

Wollheim, Richard. 1980. Art and its objects. Cambridge: Cambridge University Press.

Zimmermann, Thomas Ede. 2000. Free choice disjunction and epistemic possibility. Natural Language Semantics 8(4). 255-290.

Dilip Ninan

Department of Philosophy

Tufts University

Medford, MA, USA

dilip.ninan@tufts.edu 